\title{
High-temperature-required protein A2 as a predictive marker for response to chemotherapy and prognosis in patients with high-grade serous ovarian cancers
}

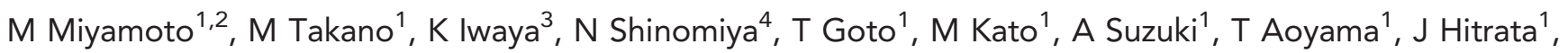
I Nagaoka ${ }^{2}, \mathrm{H} \mathrm{Tsuda}^{3}$ and K Furuya ${ }^{1}$

${ }^{1}$ Department of Obstetrics and Gynecology, National Defense Medical College Hospital, Tokorozawa, Saitama 359-8513, Japan; ${ }^{2}$ Department of Host Defense and Biochemical Research, Juntendo University Graduate School of Medicine, Tokyo 113-8431, Japan; ${ }^{3}$ Department of Basic Pathology, National Defense Medical College Hospital, Tokorozawa, Saitama 359-8513, Japan and ${ }^{4}$ Department of Molecular Biology, National Defense Medical College Hospital, Tokorozawa, Saitama 359-8513, Japan

Correction to: British Journal of Cancer (2015) 112, 739-744. doi:10.1038/bjc.2015.1; Published online 27 January 2015

Following publication of the above paper in the British Journal of Cancer, the authors noticed a mistake in the affiliations of the first author Dr M Miyamoto. The correct author affiliations are listed above. 